The element of confidence on the part of the patient is of the very greatest importance. If you have a person giving the anesthetic in whom the patient has confidence that patient is not likely to get into trouble. If the patient vomits after the operation, gastric lavage will give immediate relief in most cases.

Dr. M. B. TINkER, Baltimore-Local anesthesia I feel should be so much more extensively used than is now done. Many surgeons do not appreciate how much can be done under local anesthesia. As an example, hernia operations can be done under cocain and with so little discomfort to the patient that they say in many instances that the pain is less than at. tempts made to reduce the hernia by taxis. I would not advocate local anesthesia in all cases by any means, but I am convinced that it does save a certain number of deaths from ether pneumonia, lung embolism, heart failure and other causes which I do not believe can be avoided if general anesthesia is employed. Several factors essential to success in the use of a local anesthetic: In the first place the patient should understand the advantages of operation under local anesthesia, and want it done in this manner. If he does not want the operation done in this manner he will object to the slightest pain and you will not be successful. Another point of great importance is the necessity for taking plenty of time; you can rush local anesthesia. You have to take care to infiltrate tissues well and take time for dissection. If you do not you will cause your patient much pain and he will complain so bitterly that you can not complete the operation. A good sized preliminary dose of morphin is also of great importance, say 1-6 of a grain one hour before the operation, another 1-6 at the beginning of the operation and still another 1-6 during the operation. A good moral anesthetist at the head of the table will be of great advantage in distracting the patient's attention from the slight pains.

Dr. Whlliam J. Mayo, Rochester, Minn.-We have for years preferred ether to chloroform, even though there may be just as many deaths from ether as from chloroform. When a fatal result does occur, the patient has a much better chance to bid good-bye to his friends than if he died from chloroform anesthesia. Dr. Ochsner brought out one very excellent point, that the first thing to do in trouble from chloroform anesthesia is to force all of the chloroform out of the lungs by compressing the lungs before forcing the patient to take an inspiration. The Souchon anesthetizer is a very valuable apparatus for operations on the throat and mouth.

There is a large tendency in this country to settle things in the laboratory. This can not be done. Now, take the question of hemoglobin, and the influence which it exerts on anesthesia; the amount of hemoglobin present is not usually so important a fact as is the cause of a loss of hemoglobin. I have often operated when the hemoglobin was as low as 25 per cent., due to hemorrhage, and I have never seen an accident following such an operation. But if you do an operation on a case in which from some malignant cause the hemoglobin is reduced to 40 per cent. or 50 per cent., and the oxygen-carrying power of the patient is decreased, you stand a chance to lose the case because with cachexia the blood is poor in oxygen-carrying power. We are exceedingly afraid of low hemoglobin in cancerous disease, but I would not hesitate to perform an operation with the hemoglobin as low as 25 per cent., provided the disease is due to blood loss resulting in anemia, and provided I can reach the cause of the loss of hemoglobin.

Dr. John E. Summers, JR., Omaha-I want to call attention to the post-operative effects of anesthetics on the temperature curve. For some time I have been noticing that after prolonged operations under ether we would occasionally get a temperature curve. This used to be called "reaction," but now I have become convinced that it is due to the anesthetic. I have gotten so that I think I can recognize it, and am not alarmed.

Anybody who has lost a case on the table never forgets the anesthetic. I have had the misfortune to lose one case on the table from chloroform, and it does not make me feel any bet- ter to know that every surgeon in my vicinity has had the same experience. Death comes so suddenly that it does not give you an opportunity to protect yourself or your patient. Dr. Mayo has expressed it very nicely by saying that it does not even give the patient an opportunity to say good-bye to his physician or his friends. Recently a little child was brought into the hospital with a compound fracture of the leg. $\mathrm{He}$ was given chloroform and the fracture reduced. Just as he was coming out of the anesthetic he vomited and aspirated some potato into his trachea. I opened the trachea immediately, but the child died from asphyxiation. I think it is always better in emergency work, especially among children, to wash out the stomach prior to the administration of the anesthetic, and in our hospital where much emergency work is done, I have made this a standing order because we can not know whether the stomach is empty or full.

In the Johns Hopkins Hospital, I notice by their Bulletin, that Cushing has found by experiments with the tonometer that chloroform always reduces arterial pressure more than does ether. I think there is no doubt that we are more liable to have accidents happen if we use chloroform than if we administer ether.

Dr. E. J. Meluish, El Paso, Texas-Dr. Ochsner spoke; about using chloroform in all cases when he has a competent anesthetizer. It can be used more safely with a competent anesthetizer, but I have found from experience that no matter how chloroform is used it is more dangerous' than ether. Dr. Moore's favorable experience with chloroform is doubtless due to skilful management of the anesthesias. Within a period of eight years I saw four deaths on the table from anesthetics, three from chloroform and one from ether. The patient who died from ether was extremely apprehensive before the anesthesia-lacked the necessary confidence in the anesthetizer. When this confidence is absent the danger is greatly increased. I have had at my disposal the records of 6,000 anesthesias at a Chicago hospital. The first three thousand anesthetics were given under the supervision of skilled anesthetizers. The last three thousand were given by those much less skilled in the administration of anesthetics. There were three deaths in this series, all occurring in the second three thousand cases. When chloroform is used, dangerous symptoms are likely to develop much more rapidly than after ether administration, and in the latter condition you have a much better chance to resuscitate your patient.

Another thing is, that chloroform, being heavy, is eliminated very slowly, and it has been detectea in the sputa two or three days after the anesthesia. This neans that the patient is continuously more or less poisoned during this time. Ether is eliminated much more rapidiy.

\section{TWO CASES OF PARATYPHOID INFECTION; ONE CAUSED BY AN ABERRANT ORGANISM.}

G. BERTNARD SMITH, S.B.

(FRON THF BACTERIOLOGICAL LABORATORY, UNIVERSITY OF CHICAGO.) CHICAGO.

Cases of paratyphoid fever, both sporadic and epidemic, ${ }^{1}$ have been reported so frequently of late that perhaps some special reason should be given for calling attention to any more isolated cases. In the present instance the author believes that the bacteriologic findings are of sufficient interest to warrant a brief presentation:

CASE 1.-The patient, a man aged 58 years, entered the Presbyterian Hospital, Oct. 22, 1902. His temperature by mouth was 102.2 , and he complained of frontal headache and severe abdominal pain. The abdomen was tender everywhere, but the liver and spleen were not palpable and no rose spots were visible. November 1 the patient had two hemorrhages from the bowels. The first was slight and only a small amount of blood was cast out. About three pints of liquid blood containing some clots were discharged with the second hemor-

1. Conradi and v. Drigalski : Zelt. f. Hyg., 1903, xlil, 141. 
rhage. The abdominal distress and headache continued through the first week of November, and during this time the patient was very weak and restless and at times slightly delirious. Beginning with the second week of November he began to show definite signs of improvement, and from this time his progress was uninterrupted and he was discharged November 21 .

No rose spots were found at any time. The Widal test for typhoid was persistently negative throughout the course of the disease, even in dilutions as low as 1 to 10 .

Five cubic centimeters of blood were drawn from one of the large veins of the arm, using the usual surgical precautions for asepsis. About 3 c.c. of this blood were put into five flasks each containing 150 c.c. of sugarfree bouillon and within twenty-four hours the organism to be described was obtained in pure culture.

This organism resembles the typhoid bacillus in morphology. It shows no capsule; it is not sporogenic. It stains somewhat unevenly with anjlin dyes and is readily decolorized by Gram's method. It is motile, but is more sluggish than $B$. typhosus.

CULTURAL CHARACTERISTICS. ${ }^{2}$

Bouillon.--A uniform turbidity is shown in twentyfour hours. No scum forms even when growth continues for several days.

Agar.-A heavy white growth appears in twenty-four hours. This is moist with a raised margin and a smooth homogeneous surface. The growth is largely limited to the line of inoculation.

Litmus Milk.--No change is noticeable during the first forty-eight hours, but after the second day the reaction soon becomes strongly acid. No precipitation of casein occurs. At the end of several weeks the medium presents a deep blue color.

Gelatin.-No liquefaction occurs.

Neutral-red Agar. ${ }^{3}$-Both stab and shake cultures were used. A few gas bubbles formed, but no change occurred in the color of the media, even after several weeks.

Carbohydrates.-Glucose, saccharose, levulose, lactose, mannite, galactose and dextrin were tested in the fermentation tube. One per cent. of the carbohydrate was added to ordinary nutrient broth after removing the muscle sugar. Care was observed in every case to prevent overheating the sugar broths, and all that became darkened in heating were discarded. Gas production occurred only in the glucose broth.

\section{REPORT OF A SECOND CASE.}

A second case came to the writer's attention at the time the organism just described was being studied. This case was a private patient of Dr. James $W$. Walker and the organism was isolated by Dr. Harris of the College of Physicians and Surgeons, Chicago, and given to me for further study. This organism resembles very closely the bacillus isolated from Case 1 in its morphology and growths in bouillon, agar and gelatin.

Litmus Milk.-A slight acidity is noticed within the first twenty-four hours, but this is lost during the second or third day and the color again becomes neutral. After several weeks the medium assumes the same deep blue color described under Case 1.

Neutral-red Agar.-Gas bubbles are formed within twenty-four hours in both stab and shake cultures. The

2. Except where stated otherwise all medla were prepared ac cording to the "Proceedings Recommended for the Study of Bacteria." Reports and papers of the American Public Health Associa tion, 1898 , xxvili, p. 60 .

3. Neutral red agar was prepared by adding to neutral agar 0.5 per cent. dextrose and 1 per cent. of a 0.5 per cent. aqueous solution of neutral red (Grübler's). color is rapidly changing during this time so that only in the lower one-fifth of the medium does the color still persist. It is almost entirely reduced to a light yellow by the end of the fourth day. After the first week the color gradually returns until the original appearance is again given. This return of color seems to occur from above downward instead of from below upward, as described by Buxton.

Carbohydrates.-This organism was grown in saccharose, glucose, mannite, levulose, lactose and dextrin media, using 1 per cent. in bouillon as before. Gas was formed in each case except in the broth containing saccharose and lactose. The fermentation tests with this organism seemed to follow so closely the results described by Buxton ${ }^{4}$ with his paratyphoid bacillus and closely allied forms that a comparative study was made in the different carbohydrates with the two forms here described and Buxton's organism. The gas formed was only roughly analyzed by the use of 2 per cent. $\mathrm{NaOH}$.

PARACOLON BACILLUS FROM BUXTON'S CASE 7 .

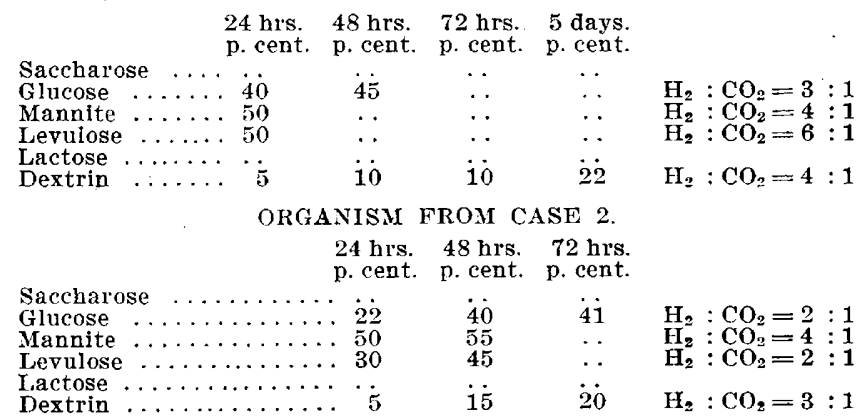

ORGANISM FROM CASE 1 .

24 hrs. 48 hrs. 72 hrs.

$\begin{array}{cccc}\text { p. cent. p. cent. p. cent. } & \text { po } & \mathrm{H}_{2}: \mathrm{CO}_{2}=5: 1\end{array}$

Glucose* $\ldots \ldots \ldots \ldots \ldots \ldots \ldots$.
No fermentation was obtained with saccharose, mannite, levuose, Iactose and dextrin.

A comparison of the tables showing the carbohydrate tests indicates a close relationship between Buxton's paratyphoid bacillus and the organism ${ }^{\bar{y}}$ isolated from Case 2. This is also shown by a comparison of the growths in litmus milk and neutral-red agar. In order to study still further this similarity a comparison was made of the agglutinative characteristics of Buxton's form and the two organisms here described.

\section{MICROSCOPIC AGGLUTINATION TESTS.}

No dilutions were made above 1 to 100 . Buxton's paracolon was tested with serum from Case 2 and agglutination was shown in dilutions as high as 1 to 50 . Serum from Case 2 was tested with Bacillus 1, and agglutination occurred in all dilutions as high as 1 to 50 . Above this, dilutions as high as 1 to 100 gave almost complete agglutination, but I was unable to decide at any time that all motility was positively lost. Serum from Case 1, and also from Case 2, was tested with typhoid and hog cholera bacilli and $B$. enteritidis with negative results in every case even in dilutions as low as 1 to 10 .

\section{MACROSCOPIC AGGLUTINATION TESTS.}

Small hemolysis test tubes were used in which the bacilli, in definite quantities, were added to a serum of the desired dilution, and after three hours' incubation the results were noted with the naked eye. The agglutination was considered positive only when complete

4. Buxton: Jour. Med. Research, 1902, viii, 201.

5. The forms isolated from Cases 1 and 2 will hereafter be referred to as Bacillus 1 and Bacillus 2 respectively. 
precipitation had occurred, leaving the liquid clear. Serum was obtained from four rabbits. Of these, one was immunized with Buxton's paratyphoid bacillus, the inoculations being continued until rapid agglutination was given at 1 to 2,000 . A second rabbit was immunized with a typhoid organism. ${ }^{\circ}$ The third rabbit was inoculated with the bacillus of hog cholera ${ }^{7}$ until complete agglutination was given at 1 to 1,000 . The fourth rabbit was inoculated with the $B$. enteritidis. ${ }^{8}$ The degree of agglutinative power reached in the serum of this rabbit was much lower than in any of the others, as the highest dilution giving positive results was 1 to 400 .

Serum from the rabbit immunized with Buxton's paratyphoid bacillus gave negative results in dilutions of 1 to 10 with the bacilli of typhoid and hog cholera and with $B$. enteritidis.

Sera from the rabbits that had been inoculated with the organisms of typhoid, hog cholera and with $B$. cnteritidis gave negative results at all dilutions with Buxton's form and with both Bacillus 1 and Bacillus 2 . Bacillus 1 , when added to Buxton's serum, was agglutinated at 1 to 100 . Above this the precipitation was never found to be complete. Bacillus 2 was completely precipitated by the Buxton serum at all dilutions as high as 1 to 2,000. No dilutions were tried above this. Using the sign + to indicate complete agglutination, these results may be tabulated as follows:

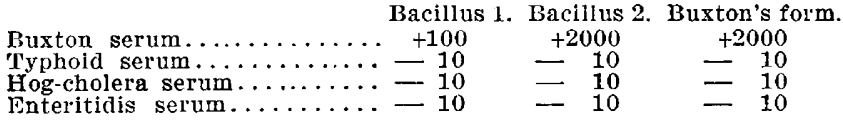

To summarize, Bacillus 2 must be regarded as an . organism which posisesses the general characteristics of the paracolons, and, from its growth in litmus milk and neutral-red agar, together with the fermentation and agglutination tests, possesses a close relationship to Buxton's form. I have not made comparisons with other members of the paratyphoid group, but from Buxton's comparative study Bacillus 2 would seem to belong to the subgroup which comprises the organism isolated by Gwyn, ${ }^{2}$ two forms described by Johnston, ${ }^{10}$ Hewlett's form, ${ }^{11}$ the bacillus Müller of Schottmïller, ${ }^{12}$ Buxton's paracolon ${ }^{13}$ and possibly Cushing's ${ }^{14}$ Bacillus $O$.

Bacillus 1, while conforming to the general tests for paratyphoid bacilli, seems to occupy rather an independent position. The clinieal history of this case is specially interesting because it shows the occurrence of two hemorrhages from the bowel. In the eighty-four cases of paratyphoid infection that have been reported, but four cases with hemorrhage have occurred. One was the case reported by Gwyn. Another, reported by Strong ${ }^{15}$ showed a complication from malarial infection. The third case was reported by $\mathrm{Hume}^{16}$ and the fourth was

6. This form was Typhoid 6 of Theobald Smith, which was originally obtained from the spleen of a typhoid patient. This serum agglutinated the typhoid bacillus in dilution of 1 to 25,000 .

7. This organism is one obtained foom V. A. Moore of the New Iork State Veterinary College, Cornell University.

8. This organism is from the stock cultures of the university laboratory, and possesses the typical cultural characteristics of $B$. enteritidis. The source is unknown.

9. Gwyn: Johns Hopkins Hosp. Bull., 1898, ix, 54.

10. Johnston: Amer. Jour. Med. Sci., 1902, cxxxv, 187.

11. Hewlett: Ibid., 1002, exxiv, 200.

12. Schottmiller: Deut. med. Woch., 1900, xxvi, 511.

13. Buxton \& Coleman: Amer. Jour. of Med. Sciences, 1902, exxiii, 976.

14. Cushing: Johns Hopkins Hosp. Bull., 1900, x1, 156

15. Strong: Johns Hopkins Hosp. Bull, 1902, xiil, 107. 385.

16. Hume: Report of the Thompson Yates Laboratory, 1902, iv, one of an epidemic of fourteen cases reported by De Feyfer and Kayser. ${ }^{17}$

In morphology, Bacillus 1 shows no important points of difference from the rest of the paratyphoid bacilli which have been described, but in its special cultural characteristics it presents very marked individuality. It develops a strongly acid reaction in litmus milk, which does not, however, cause a coagulation of the casein and which changes to the typical alkaline state, although very slowly. No reduction in the color of the neutralred agar occurs, which would seem to point toward a relationship with the typhoid bacillus.

The absence of fermentation, except in the glucose media, shows a particularly wide departure from the common type of paratyphoid organism studied by Buxton.

I am indebted to Dr. E. O. Jordan for many valuable suggestions and criticisms during the progress of this work, and to Dr. B. W. Sippy, who was the attending physician to the first case I have reported, for favors received at the hospital.

\section{Glinical Reporte.}

\section{A CASE OF PARATYPHOID.}

\section{JAMES W. WALKER, M.D.}

Adjunct Professor of Medicine, College of Physicians and Surgeons. CHICAgo.

Until the symptomatology of the various forms of paratyphoid has been tabulated more completely than at present, apology for publishing clinical cases in detail should be superfluous. For the working out of the bacteriology and, therefore, for the establishment of the diagnosis, I am indebted to Mr. G. Bertnard Smith, who publishes his report separately in this number of The Joursal, and to Dr. Fred G. Harris, pathologist of Cook County Hospital, who began the culture.

Dr. H. M. R.; aged 30; Jewish; family history negative. Eleven years ago was ill for ten days with what was said to be typhoid, but this is doubtful. Has not been out of Chicago for years. Present illness began about Sept. 10, 1902, with malaise, chilly sensations, headache, anorexia, free perspiration and slight temperature, with which symptoms patient went about his professional duties for a week, and on September 17 went to bed with what he and I presumed was ordinary typhoid fever. Pulse was then 80 ; physical examination negative, and it may be stated here to dispose of the matter, that at no time was the spleen palpable, there were never any rose spots, no tympanites, no diarrhea, no abdominal tenderness, in short, no abdominal symptoms nor signs. There was never any cough, nor was the tongue ever dry; patient was never unconscious, though there was some stupor during the first days in bed. From the beginning the chills (see temperature chart), perspiration and fever were the dominant symptoms, and led to a repeated search for the Plasmodium malarice, which was fruitless.

September 22 there was nausea and vomiting. September 23 , very restless and uncomfortable. Second heart tone duplicated, pulse 130, dyspnea evident when talking, no râles nor cough, complained of diffuse pain throughout the chest. September 24, numbness of both little fingers, severe chills, albumin in the urine.

September 25, (1) The repeated severe chills, with absence of the plasmodium, and despite the liberal use of quinin; (2) the drenching sweats, (3) the diffuse chest pain, (4) the increasing dyspnea, (5) the absence of the Widal and Ehrlich reactions, (6) absence of all abdominal symptoms and signs, (7) the very irregular temperature, and (8) the general evidence of profound toxemia in the absence of a discoverable local lesion, strongly inclined me to think the case one of acute miliary tuberculosis.

17. De Feyfer and Kayser: Münch. med. Woch., 1902, xlix, 1692. 